\title{
Consensus guidelines for the safe prescription and administration of oral bowel-cleansing agents
}

\author{
Andrew Connor, ${ }^{1}$ Damian Tolan, ${ }^{2}$ Stephen Hughes, ${ }^{3}$ Nick Carr, ${ }^{4}$ Charles Tomson ${ }^{3}$
}

- Additional appendices are published online only. To view these files please visit the journal online (http://dx.doi.org/ 10.1136/gutjnl-2011-300861).

${ }^{1} \mathrm{SpR}$, Dorset County Hospital, Dorchester, UK

${ }^{2}$ St James' University Hospital Leeds, UK

${ }^{3}$ Southmead Hospital, Bristol, UK

${ }^{4}$ Singleton Hospital, Swansea, UK

\section{Correspondence to}

Dr Stephen Hughes, 43

Kellaway Avenue, Bristol BS6

7XS, UK; steve.hughes@nbt.

nhs.uk

Accepted 2 May 2012

Published Online First

26 July 2012

\section{ABSTRACT}

Oral bowel-cleansing preparations are used before colonic surgery and endoscopic and radiological assessment of the intestine to minimise faecal contamination. In February 2009, the UK National Patient Safety Agency issued a Rapid Response Report highlighting the potential risk of harm associated with the use of these preparations and instructing local NHS Trusts to implement safeguards to reduce this risk. This guidance has been prepared to help NHS Trusts to respond to these concerns, as the risk of complications is influenced by both individual patient risk factors and the choice of bowel preparation, for which definitive guidance was not previously available. This document provides an outline of the different available oral bowelcleansing agents and the complications that may arise. This is followed by recommendations for their use in different patient groups and circumstances. The recommendations are based on consensus between the authors, each of whom circulated drafts to members of their specialist society. The evidence for these recommendations has been assessed using the modified GRADE system. The recommendations cover the choice, administration and complications (relative and absolute) of the different oral bowel-cleansing agents, with specific guidance provided for different patient groups.

\section{INTRODUCTION}

Oral bowel-cleansing preparations are used before colonic surgery and endoscopic and radiological assessment of large and small intestine to minimise faecal contamination. In general, these preparations are safe and well tolerated. However, in February 2009, the UK National Patient Safety Agency (NPSA) issued a Rapid Response Report alerting healthcare providers to the potential risk of harm associated with the use of oral bowel-cleansing preparations and reporting one death and 218 patient safety incidents occurring over a 5 -year period. ${ }^{1}$ Six per cent of the 218 patient safety incidents resulted in moderate harm and one patient death was reported. These data are derived from a voluntary reporting system and, as such, are subject to bias: a proportion of incidents will not be reported, and those that are reported may underestimate severity (if reported before the full patient outcome is known). The majority of these incidents were reported as relating to the administration $(56 \%)$ and prescription $(21 \%)$ of the oral bowel-cleansing agents. The manifestations of these patient safety incidents included harm as a result of prescription of bowel preparation to patients in whom there was a definite contraindication (eg, presence of ileostomy, bowel obstruc- tion) or renal failure as a result of phosphate nephropathy, complications of hypovolaemia and electrolyte disturbances including hypokalaemia, hyponatraemia and hypermagnesaemia. Although there are no reliable estimates of the frequency of each of these complications, it is reasonable to put systems in place to reduce the risk of complications, so long as this response is proportionate and does not greatly add to the complexity or cost of investigation.

\section{SCOPE}

The NPSA Report instructed NHS Trusts that safeguards should be implemented at a local level to reduce this risk, and specifically required that all NHS Trusts ensure that a clinical assessment of each patient for contraindications and risks occurs, that the use of a bowel-cleansing preparation is authorised by a clinician, that an explanation on the safe use of the preparation is provided to the patient, and that a safe system exists for the supply of the preparation for each patient. This guidance has been prepared to help in the first of these recommendations relating to clinical assessment. We believe that guidelines are necessary because the risk of complications depends on the choice of bowel preparation and on risk factors present in the individual patient and there has been to date no definitive guidance on which preparation to use for which patients. These guidelines are therefore aimed at a UK audience but may have relevance to other countries. They are intended to be of benefit to those services prescribing and administering oral bowel-cleansing agents and to those clinicians referring patients for investigations and procedures that may require their use.

The guidelines do not include recommendations on incorporation of prescription of bowel-cleansing agents in the request for investigation, nor do they cover the use of oral bowel-cleansing agents in children or in pregnancy. The guidelines do not cover preparation for radiological or endoscopic examination of the small bowel.

These guidelines do not attempt to address the thorny issue of which bowel-cleansing agent is the most effective or which regimen for administration is most useful. It does not cover scoring systems to assess the efficacy of the preparation, split dosing or other attempts to improve the quality of the bowel cleansing. Although these issues are crucial to successful bowel examination, they are beyond the scope of this document.

Guidelines for bowel preparation before colonoscopy are already in existence, but they do not adequately address the risks identified by the NPSA. $^{2}$ 


\section{METHODOLOGY}

The authors were put forward by their specialist societies to produce an initial draft. One author (AC) prepared an initial document (April 2009). This was informed by an exhaustive literature review. A formal systematic review was not undertaken, as the question that these guidelines seek to answer (how best to prescribe and administer oral bowel-cleansing agents, within different patient populations, while minimising the differing risks associated with the various preparations) was considered to be more complex than might effectively be answered through systematic literature review.

The initial document was reviewed by the other authors, revised individually, and a consensus draft agreed upon (June 2009). This was then circulated to the appropriate committees for review within each specialist society. The following committees were consulted: the Endoscopy Committee of the British Society of Gastroenterology; the Clinical Practice Guidelines Committee of the Renal Association; the British Society of Gastrointestinal and Abdominal Radiologists Committee and the Board of the Faculty of Clinical Radiology (for the Royal College of Radiologists); and the Clinical Services Committee of the Association for Coloproctology of Great Britain and Ireland (for The Royal College of Surgeons). The revised guidelines were then posted on the respective websites of the specialist societies as draft guidelines for further comment (early 2010), thereby allowing the guidelines to be available as interim guidance for members. Further revisions were made on the basis of feedback from members and after review by the Clinical Services and Standards Committee of the British Society of Gastroenterologists (late 2010), before submission of the guidelines for publication (January 2011).

In order to provide early draft guidance on the websites of the contributing groups according to the timescale imposed by the NPSA (requiring implementation of the recommendations in the Rapid Response Report by 7 September 2009), we have not performed a systematic review nor adhered in full to the guideline development methodology recommended by the National Institute for Health and Clinical Excellence (NICE). There was no representation from patient groups nor from the Pharmaceutical Industry. The companies that market the products discussed have not been consulted for their views and some of our recommendations go beyond the summary of product characteristics. The evidence for these recommendations has been assessed by the authors using the modified GRADE system. $^{3} 4$ The modified GRADE system first defines the strength of the recommendations of guideline authors. Expert recommendations are graded as 'strong' (grade 1) or 'weak' (grade 2) balanced by benefits and risks, burden and cost. Second, the quality or level of evidence upon which the recommendation is based is designated as high (grade A), moderate (grade B), low (grade C) or very low (grade D), depending on study design and consistency of results. Grades of recommendation and quality of evidence may therefore range from $1 \mathrm{~A}$ to $2 \mathrm{D}$ (see online appendix 1). We hope that NICE will develop guidelines to cover this topic in the near future.

\section{BACKGROUND}

\section{Bowel-cleansing agents available for use}

A number of different oral bowel-cleansing agents are currently available in the UK, including the following.

- Citrafleet (De Witt, Warrington, UK); sodium picosulphate and magnesium citrate

- Citramag (Sanochemia, Bristol, UK); magnesium carbonate and citric acid
- Fleet Phospho-Soda (De Witt); sodium dihydrogen phosphate dehydrate and disodium phosphate dodecahydrate

- Klean Prep (Norgine, Uxbridge, UK); polyethylene glycol

- Moviprep (Norgine); polyethylene glycol

- Picolax (Ferring, West Drayton, UK); sodium picosulphate and magnesium citrate

The ideal oral bowel-cleansing agent would be convenient to administer, well tolerated, effective in cleansing, with an acceptable side-effect profile. No single agent is ideal in all clinical scenarios, and research into the ideal agent (or combination) continues. The different oral bowel-cleansing agents available in the UK are summarised in online appendix 2.

Polyethylene glycols (also known as PEG or macrogols) are nonabsorbable iso-osmotic solutions, which pass through the bowel without net absorption or secretion. Significant fluid and electrolyte shifts are therefore attenuated. Moviprep contains $100 \mathrm{~g}$ polyethylene glycol, while Klean Prep contains 69 g. Both Moviprep and Klean Prep contain a balanced electrolyte solution, which reduces associated fluid and electrolyte disturbances. Moviprep also contains ascorbic acid, which contributes to the cathartic effect of the preparation. The preparations must be diluted in large volumes of water (up to 4 litres) to achieve the desired cathartic effect, and often have an unpalatable taste (despite flavourings). Compliance is better with divided-dose regimens (eg, the initial $2-3$ litres on the night before the procedure and the remaining 1-2 litres the following morning). ${ }^{6}$ Not all of the ingested water stays within the gut lumen; absorption of water can therefore lead to water intoxication in predisposed patients.

Conversely, oral sodium phosphate preparations are hyperosmotic and promote colonic evacuation by drawing large volumes of water into the colon (1-1.8 litres of water per $45 \mathrm{ml}$ of preparation). ${ }^{7}$ They are typically diluted in much smaller volumes of water than the polyethylene glycols $(\sim 250 \mathrm{ml})$.

Sodium phosphate preparations have been compared with polyethylene glycols in numerous studies and have generally been found to be safe, equally effective and consistently better tolerated. $^{8-12}$ Two meta-analyses are of note. One meta-analysis of eight controlled trials concluded that, while an 'excellent' preparation was more likely with sodium phosphate preparations, 'adequate' preparation was equally likely with sodium phosphate or polyethylene glycol preparations. 9 More recently, data regarding the quality of bowel preparation from 104 randomised controlled trials comparing bowel preparation regimens for colonoscopy were pooled in multiple meta-analyses exploring a range of inclusion criteria. ${ }^{13}$ Overall, no significant difference in the quality of bowel cleansing achieved with sodium phosphate and polyethylene glycol preparations was identified, although the latter were comparatively more efficacious in preparing the proximal bowel and also when previousday regimens were used.

Picosulphate is a prodrug that is metabolised within the bowel lumen to a stimulant that promotes peristalsis. It is often combined with magnesium salts (eg, in Picolax or Citrafleet), which act synergistically through their osmotic effects. ${ }^{14}{ }^{15} \mathrm{~A}$ dose sufficient to provide adequate bowel cleansing is usually diluted in a total of $300 \mathrm{ml}$ of water. Data on efficacy of cleansing are mixed when compared with other agents. ${ }^{16-21}$ It remains widely used for bowel preparation for radiological procedures. $^{22-25}$ One study of 72 patients over 70 years of age found that good overall bowel preparation was achieved in $88 \%$ of patients receiving Picolax before CT colonography. ${ }^{26}$

Preparations of magnesium carbonate with citric acid, such as Citramag, are osmotic saline agents that require only $200 \mathrm{ml}$ of water as a diluent. Magnesium salts are well tolerated and 
effective, and have been reported to be used to prepare the bowel in one in every three colonoscopies undertaken in the UK. ${ }^{27}$

Some types of bowel preparation leave a significant amount of watery residue in the gut lumen, which is not a problem for endoscopic or surgical procedures. However, this may interfere with mucosal visualisation at CT colonography and barium enema and these laxatives are usually avoided for radiological imaging. Picolax produces the 'driest' bowel, Citramag is intermediate, and polyethylene glycol preparations leave the highest amount of watery residue. The choice of agent therefore depends to some extent on which procedure the patient is being prepared for.

Bioavailability of some medications may be affected by bowel cleansing (eg, oral contraceptive pill). There is no evidence relating to bioavailability of immunosuppressive agents. Oral iron should be stopped at least 5 days before colonoscopy, as it forms an adherent residue that interferes with mucosal visualisation.

Diabetic glycaemic control, particularly in patients with type 1 diabetes, can be problematic during the period of dietary restriction, requiring individualised advice from local diabetic specialists. Admission for intravenous glucose and insulin may be required in a small number of cases.

Preparations vary in the requirement for dietary restrictions; most require that a clear liquid or low-residue diet should be followed for the $24 \mathrm{~h}$ or longer before the procedure, but with Fleet Phospho-Soda it is only necessary to avoid solid food during the dosing period.

Combinations of different bowel-cleansing agents (eg, Picolax and Klean Prep or combinations of senna granules with Citramag) are used in some centres ${ }^{28}$; these regimens are beyond the scope of these guidelines.

\section{Complications from bowel-cleansing agents}

When administered correctly, all of the preparations listed have been demonstrated to be safe for use in healthy individuals without significant comorbidity, and to effect adequate bowel cleansing. ${ }^{8} 29-32$ However, as hypertonic solutions, sodium phosphate preparations can cause major fluid and electrolyte shifts, and should generally be considered second-line agents that should only be prescribed to patients without other comorbidities (in particular, these preparations should be avoided in those with chronic kidney disease, congestive cardiac failure, liver failure, hypertension or patients taking renin-angiotensin blockers or diuretics).

Current practice for elective procedures is typically for patients to self-medicate with oral bowel-cleansing agents at home, often received through the post without formal screening of their comorbidities, medications or hydration state. While the practice of self-medication at home should remain feasible for the majority of patients, it is clear that a screening process is necessary to ensure that patients at risk of harm from oral bowel-cleansing agents are identified and prepared appropriately.

\section{Hypovolaemia}

Patients receiving oral bowel-cleansing agents are at risk of developing the complications of hypovolaemia and intravascular volume depletion-including syncope, myocardial ischaemia and acute kidney injury secondary to acute tubular necrosis. This risk is likely to be greatest with sodium phosphate preparations, but also exists with sodium picosulphate (although a recent study of 72 patients receiving Picolax before CT colonoscopy revealed no significant changes in estimated glomerular filtration rate $\left.(\mathrm{GFR})^{26}\right)$; the risk of hypovolaemia is least with polyethylene glycol preparations.

\section{Hypokalaemia}

The frequency with which hypokalaemia occurs after oral bowel-cleansing preparations is variable. In studies of patients receiving sodium phosphate preparations, hypokalaemia has been reported to occur in $56 \%$ of older inpatients and $26 \%$ of unselected patients. ${ }^{33} 34$ A retrospective study found the incidence of hypokalaemia $(<3.0 \mathrm{mmol} / \mathrm{l})$ in patients administered polyethylene glycol preparations before colonoscopy to be $9.6 \%{ }^{35}$ Hypokalaemia can occur for two reasons after bowel preparation: increased gastrointestinal loss of secreted potassium complicating the use of hyperosmotic and stimulant preparations, and, with the use of sodium phosphate, increased urinary loss as a result of hyperphosphaturia. ${ }^{36}$ Co-administration of a carbohydrate-electrolyte solution with sodium phosphate has been reported to reduce the risk of hypokalaemia. ${ }^{37}$

\section{Hyponatraemia}

The ingestion of large volumes of water, particularly in the context of reduced free water clearance, also predisposes patients to hyponatraemia (a risk that was highlighted specifically in the NPSA Rapid Response Report). Polyethylene glycol preparations involve the ingestion of up to 4 litres of water, but are designed to be isotonic. The risk of hyponatraemia is probably highest when excessively large volumes of water are ingested (as a result of overzealous adherence to advice to 'drink plenty of fluids') to offset water loss into the colon caused by oral sodium phosphate and sodium picosulphate preparations. ${ }^{38}$ Hyponatraemia has also been reported after use of polyethylene glycols, but is rare. ${ }^{39}$ Reports of hyponatraemia occurring with magnesium-based agents are very rare. ${ }^{40}$

\section{Phosphate nephropathy}

Acute phosphate nephropathy is an increasingly reported but underdiagnosed cause of chronic kidney disease, ${ }^{41}{ }^{42}$ which may occur in up to 1 in 1000 patients who receive sodium phosphate preparations. $^{41}$ Oral sodium phosphate preparations provoke a transient mild hyperphosphataemia, ${ }^{8}$ which is most profound in older subjects. ${ }^{43}$ This is rarely associated with untoward events and may reflect the normal reduction in GFR with advancing age. For this reason, the recommendations in this document are based on GFR and not on age. However, other factors that promote hyperphosphataemia predispose patients to acute phosphate nephropathy, such as inappropriate phosphate dosing, increased bowel transit time, and a reduced ability to excrete a phosphate load (such as renal impairment). ${ }^{44}$ Factors promoting tubular precipitation of calcium phosphate also predispose to acute phosphate nephropathy and include inadequate hydration during phosphate administration, hypertension with arteriosclerosis, and medications including non-steroidal anti-inflammatory drugs (NSAIDs), diuretics and reninangiotensin inhibitors. ${ }^{42}$ Heart failure, cirrhosis and advancing age are additional risk factors. ${ }^{45} 46$

Recent concerns over acute phosphate nephropathy are reflected in changes made to the availability of oral sodium phosphate preparations by the US Food and Drug Administration. These preparations are no longer available as over-thecounter medications for oral bowel cleansing, and those sodium phosphate preparations that are available as over-the-counter laxatives now carry a boxed warning. ${ }^{47}$ 


\section{Hypocalcaemia}

Hypocalcaemia is a direct result of hyperphosphataemia and has been reported to occur in $58 \%$ of patients who receive oral sodium phosphate. ${ }^{33}$ Hypoparathyroidism is a risk factor for severe hypocalcaemia in this situation. ${ }^{36}$

\section{Hypernatraemia}

Hypernatraemia is uncommon, but can occur as a result of the sodium load in oral sodium phosphate preparations in combination with inadequate water intake. ${ }^{36}$

\section{Hypermagnesaemia}

Those preparations containing magnesium salts (Picolax, Citrafleet and Citramag) can cause a transient rise in serum magnesium levels. They present a risk of hypermagnesaemia in patients with chronic kidney disease and can potentially lead to magnesium toxicity. A small number of such cases have been reported. ${ }^{48-50}$ The respective summary of product characteristics for each of the individual magnesium-based preparations advocates the use of alternative preparations in patients with 'severely reduced renal function'. In a recent study of 72 older patients with a mean estimated GFR of $60.8 \mathrm{ml} / \mathrm{min}$ per $1.73 \mathrm{~m}^{2}$ and receiving sodium picosulphate with magnesium citrate (Picolax) before CT colonoscopy, serum magnesium levels were measured before and after the administration of Picolax in 14 patients. $^{26}$ Although without clinical sequelae in this study, three patients experienced an increase in serum magnesium in excess of $0.25 \mathrm{mmol} / \mathrm{l}$.

\section{Is a bowel-cleansing agent required?}

Oral bowel-cleansing agents have traditionally been prescribed (predominantly on the basis of observational data and expert opinion) before elective colorectal surgery in an effort to reduce the likelihood of surgical complications arising from anastomotic leakage. However, opinion is increasingly divided on the merits of bowel preparation in this context. There is an increasing body of evidence to suggest that bowel preparation is not required for most procedures. Two recent trials are particularly noteworthy. First, in a trial randomising over 1300 patients, Jung et al found no appreciable difference in clinical anastomotic leaks and intra-abdominal abscesses between those patients receiving bowel preparation and those receiving no bowel preparation (2.6\% vs $4.3 \%$, effect difference $1.7 \%$, $95 \%$ CI 0.7 to 2.7$).{ }^{51}$ Similar conclusions were reached by Contant et al, who randomised 1431 patients undergoing elective colorectal surgery to receive an oral bowel-cleansing agent (polyethylene glycol or oral sodium phosphate) or no bowel preparation. ${ }^{52}$ While the rate of intra-abdominal abscesses was slightly higher in the group not receiving bowel preparation (4.7\% vs $2.2 \%$, $\mathrm{p}=0.02)$, the general incidence was low. All other end points (mortality, length of hospital stay, re-intervention rate) were similar among the two groups.

At present, patients who undergo abdominoperineal excision of the rectum, right hemicolectomy, total proctocolectomy and ileoanal pouches are generally not prescribed oral bowelcleansing agents. However, oral bowel-cleansing agents are used more widely in patients undergoing anterior resection and leftsided resections. Postoperative rapid recovery programmes are being increasingly used and usually avoid bowel preparation.

In patients requiring bowel investigation, with comorbidity that may increase the risk of complications from bowel preparation, it is worth considering the role of investigations that require minimal or no formal bowel purgation. CT colonography with faecal tagging is an area of growing clinical interest and research, using iodinated or barium-based contrast to mark faeces in the colon. It is an effective method of diagnosing and excluding colon cancer and other colonic diseases and potentially avoids the complications of bowel preparation. CT colonography is likely to have an increasingly prominent role in the future, particularly if bowel purgation can be avoided.

Gastrografin is commonly used for small-bowel studies (eg, the investigation of postoperative ileus) and sometimes for CT colonography. It is hyperosmolar and, when used undiluted and/ or with high doses, may cause an osmotic diarrhoea. Recommendations on its use are beyond the scope of these guidelines, but clinicians should be aware of the potential risk of causing hypovolaemia.

Finally, these guidelines are intended to reduce the risk of complications from the use of oral bowel-cleansing agents, but they do not address every situation and are not a substitute for sound clinical judgement.

\section{AN INDEX OF THE RECOMMENDATIONS}

1. Absolute contraindications to the use of oral bowel-cleansing agents.

2. The choice of oral bowel-cleansing agent.

3. The administration of oral bowel-cleansing agents.

4. Relative contraindications: circumstances in which the choice of a particular oral bowel-cleansing agent or administration protocol may confer significant benefits.

4.1. Chronic kidney disease

4.2. Haemodialysis patients

4.3. Peritoneal dialysis patients

4.4. Renal transplant patients

4.5. Congestive cardiac failure

4.6. Liver cirrhosis and/or ascites

4.7. Patients taking particular medications

Renin-angiotensin blockers

Diuretics

NSAIDs

Medications known to induce the syndrome of inappropriate antidiuretic hormone secretion

5. Areas in which further research is needed.

\section{RECOMMENDATIONS}

The following conditions are absolute contraindications to the use of all oral bowel-cleansing preparations.

- Gastrointestinal obstruction or perforation, ileus or gastric retention

- Severe acute inflammatory bowel disease or toxic megacolon

- Reduced levels of consciousness

- Hypersensitivity to any of the ingredients

- Inability to swallow without aspiration (in this situation a nasogastric tube may be used for administration)

- Ileostomy

Evidence: grade 1D

\section{The choice of oral bowel-cleansing agent}

The choice of oral bowel-cleansing agent requires consideration of the particular indication, the individual recipient, and the advantages and disadvantages (eg, tolerability, efficacy and potential adverse effects) of the different preparations available. Although oral sodium phosphate preparations are often well tolerated and effective, and are generally safe, ${ }^{8-12}$ the following recommendations are intended to reflect first a concern that adverse outcomes of greater severity may occur more commonly 
with these agents, and second that particular patient groups appear to be more at risk of such complications.

Magnesium salt preparations are relatively contraindicated in patients with stage 4 and 5 chronic kidney disease (see online appendix 3 for the definition of chronic kidney disease) (evidence: grade 2D).

Sodium picosulphate preparations should be used with caution in patients at risk of, or suffering from, hypovolaemia, including those patients taking high-dose diuretics, those with congestive cardiac failure and advanced cirrhosis, and those with chronic kidney disease (evidence: grade 1C).

The use of oral sodium phosphate preparations is strongly discouraged in patients with chronic kidney disease, pre-existing electrolyte disturbances, congestive cardiac failure or cirrhosis, or with a history of hypertension (evidence: grade 1C).

The use of oral sodium phosphate preparations in otherwise healthy patients is currently acceptable in cases where sodium picosulphate, magnesium salts and polyethylene glycols have proven ineffective or intolerable (evidence: grade $2 \mathrm{C}$ ).

\section{The administration of oral bowel-cleansing agents}

The appropriate doses of oral bowel-cleansing preparations should not be exceeded (evidence: grade 1C)

Where sodium phosphate preparations are prescribed, modification of the standard dose (two $45 \mathrm{ml}$ doses $9-12 \mathrm{~h}$ apart) to a $45 \mathrm{ml}$ dose followed by a $30 \mathrm{ml}$ dose should be considered (evidence: grade $1 \mathrm{C}$ ). The latter regimen provides equally effective bowel cleansing, but a significantly lower serum phosphate level. ${ }^{53}$ Furthermore, increasing the interval between doses to $24 \mathrm{~h}$ reduces the incidence of clinically relevant hyperphosphataemia (>2.1 mmol/l) without compromising efficacy. ${ }^{54}$ This lengthened preparation process may, however, be more disruptive and less acceptable to patients. Therefore, when sodium phosphate preparations are being administered, a regimen of a $45 \mathrm{ml}$ dose followed by a $30 \mathrm{ml}$ dose $24 \mathrm{~h}$ later should be considered (evidence: grade $2 \mathrm{C}$ ).

The period of bowel cleansing should not normally exceed $24 \mathrm{~h}$ (evidence: grade 1C)

To improve both tolerability and efficacy, consideration should be given to splitting the dose of oral bowel-cleansing agent over $12 \mathrm{~h}$ when polyethylene glycol preparations are used. This may not be necessary when Moviprep is used because of the lower volume of fluid ingested (evidence: grade $2 \mathrm{~B}$ ).

\section{Hypovolaemia must be corrected before administration of oral} bowel-cleansing preparations (evidence: grade 1C)

Patients with comorbidities indicating a predisposition to hypovolaemia should be assessed before starting administration of oral bowel-cleansing agents. Patients at particular risk of hypovolaemia include (but are not limited to) those with chronic or severe diarrhoea, chronic vomiting, dysphagia and persistent hyperglycaemia and those taking high-dose diuretics (see below). Admission to hospital for prehydration may be necessary (evidence: grade 2D).

Where intravenous fluid replacement is undertaken, isotonic fluid (eg, Hartmann's solution) may be preferable ${ }^{55}$ (evidence: grade $2 \mathrm{D})$.

Hypovolaemia must be prevented during administration of oral bowel-cleansing preparations (evidence: grade 1C)

Patients should receive clear instructions regarding oral fluid intake (including an appropriate volume) and these instructions should also be provided in writing (evidence: grade 1D).
Some patients receiving polyethylene glycol may achieve adequate bowel preparation without consuming the full 4 litres of fluid that are generally suggested. ${ }^{56}$ It is reasonable to advise patients to discontinue the oral bowel-cleansing agent if their bowel motions become watery and clear. Intake of other fluids should, however, continue until $2 \mathrm{~h}$ before the procedure (evidence: grade $2 \mathrm{C}$ ).

Isotonic electrolyte oral rehydration solutions may be of benefit, $^{37} 57$ and should be considered in place of high water intake for patients at risk of hyponatraemia being prescribed sodium picosulphate or sodium phosphate (evidence: grade $2 \mathrm{C}$ ).

Admission for intravenous fluid replacement should be considered in all patients who may be unable to maintain adequate oral intake at home (eg, older patients and those with reduced mobility) (evidence: grade $1 \mathrm{C}$ ).

If no recent measurement of kidney function is available (within 3 months), kidney function should be measured (using an estimated GFR from serum creatinine concentration) as recommended by NICE, in patients with any of the known conditions listed below

- Diabetes

- Hypertension

- Cardiovascular disease (ischaemic heart disease, chronic heart failure, peripheral vascular disease and cerebral vascular disease)

- Structural renal tract disease, renal calculi or prostatic hypertrophy

- Multisystem diseases with potential kidney involvement (eg, systemic lupus erythematosus)

- Family history of stage 5 chronic kidney disease or hereditary kidney disease

- Haematuria or proteinuria

- (See NICE CG73 Chronic Kidney Disease)

- Evidence: grade 1C

Where a polyethylene glycol preparation is used, renal function tests remain preferable if the above conditions are present, although polyethylene glycol preparations can be used in patients with renal impairment. The renal function tests and electrolytes may, however, be useful in assessing hydration status.

\section{Advice regarding regular medications}

Patients should be advised that their regular oral medications should not be taken $1 \mathrm{~h}$ before or after administration of bowelcleansing preparations because of the possibility of impaired absorption (evidence: grade 1C).

Patients taking the oral contraceptive pill should be advised to take alternative precautions during the week after the administration of the oral bowel-cleansing agent (evidence: grade 1C).

Patients in whom the possibility of a reduction in the absorption of their regular medications may prove catastrophic (eg, patients taking immunosuppression for transplants) may require admission for the administration of intravenous medications (evidence: grade 2D).

Patients with diabetes mellitus receiving treatment with insulin will also require specific advice, which should be agreed locally so as to be consistent with local practice and guidance for management of diabetes mellitus while 'nil by mouth' or on reduced oral intake.

Relative contraindications to the use of oral bowel-cleansing preparations: circumstances in which the choice of a particular oral bowel-cleansing agent or administration protocol may confer significant benefits

Polyethylene glycol is safer than sodium phosphate preparations for patients with electrolyte or fluid imbalances in conditions 
such as chronic kidney disease, congestive heart failure and liver failure.

Moviprep requires a smaller total volume of fluid (2 litres) to be consumed than Klean Prep (4 litres) and may be preferable in patients in whom the ability to ingest high volumes of fluid causes concern.

\section{Chronic kidney disease}

Knowledge of an individual's excretory renal function is an important consideration when identifying the most appropriate oral bowel-cleansing preparation. Pre-existing chronic kidney disease (sometimes unrecognised) is the single most important factor in the development of acute phosphate nephropathy in patients receiving oral sodium phosphate preparations.

Patients with pre-existing electrolyte imbalances should not receive oral sodium phosphate preparations (evidence: grade $1 \mathrm{C}$ ).

For patients with early chronic kidney disease (stages 1-3 (see appendix 3)), polyethylene glycols, Picolax/Citrafleet and Citramag are all acceptable oral bowel-cleansing agents. It is better to avoid sodium phosphate (evidence: grade 1C).

Patients with stage 3, 4 or 5 chronic kidney disease (an estimated GFR $<60 \mathrm{ml} / \mathrm{min} / 1.73 \mathrm{~m}^{2}$ ) should not receive oral sodium phosphate preparations (evidence: grade 1C).

Polyethylene glycol preparations may be preferable for those patients with stage 4 or 5 chronic kidney disease, who are not receiving dialysis, and who are expected to be able to tolerate the ingestion of the larger volumes of fluid required with these agents. Moviprep requires a smaller total volume of fluid (2 litres) to be consumed than Klean Prep (4 litres) and may be preferable in these patients (evidence: grade 1D).

In patients with stage 4 chronic kidney disease, the use of Picolax/Citrafleet or Citramag is associated with a small risk of magnesium accumulation and should therefore be reserved for those patients likely to be unable to tolerate the ingestion of the volume of fluid required to administer polyethylene glycol preparations (evidence: grade 2D).

In patients with stage 5 chronic kidney disease, who are not receiving haemodialysis, the use of Picolax/Citrafleet is associated with a small risk of magnesium accumulation and should therefore be reserved for those patients likely to be unable to tolerate the ingestion of the volume of fluid required to administer polyethylene glycol preparations (evidence: grade $2 \mathrm{D})$.

Owing to the possibility of magnesium accumulation, the use of Citramag should be avoided in patients with stage 5 chronic kidney disease who are not receiving haemodialysis (evidence: grade $1 \mathrm{D})$.

It should be noted that Klean Prep is currently the only oral bowel-cleansing agent available in the UK that is not contraindicated in chronic kidney disease in the summary of product characteristics.

\section{Chronic haemodialysis}

Although acute kidney injury is rarely a concern in these patients, the possibility of intravascular depletion secondary to oral bowel-cleansing agents has other implications in patients receiving chronic haemodialysis. First, there is a risk of dialysis access thrombosis in patients dialysing through arteriovenous fistulae or polytetrafluoroethylene (PTFE) grafts, where a period of intravascular depletion causes hypotension. Second, the combination of dialysis (which is itself often associated with significant fluid and electrolyte shifts) and administration of oral bowel-cleansing agents may provoke more profound hypovolaemia than would otherwise occur. Furthermore, the signifi- cant oral fluid intake required with polyethylene glycol preparations may provoke fluid overload in anuric patients. For these reasons, each case should be considered on an individual basis, and the timing of dialysis sessions should be tailored to the situation. Admission to hospital for coordination and overseeing of dialysis prescription and administration of oral bowelcleansing agents may be necessary for some patients receiving chronic haemodialysis (evidence: grade 2D).

Although contraindicated in stage 4 and 5 chronic kidney disease in predialysis patients, sodium picosulphate and magnesium salts can be used safely as oral bowel-cleansing agents in patients receiving haemodialysis (evidence: grade 2D).

\section{Peritoneal dialysis}

Peritoneal dialysis is generally associated with less significant fluid shifts than haemodialysis. Admission to hospital for administration of oral bowel-cleansing agents is therefore less likely to be necessary for the majority of patients undergoing peritoneal dialysis. However, a small proportion of these patients have a small but important degree of residual native renal function. This must be assessed on an individual basis. Measures to avoid significant fluid shifts and possible intravascular volume depletion are therefore important in this group. Admission to hospital for overseeing of administration of oral bowel-cleansing agents should be considered in those considered to have important residual renal function (evidence: grade 2D).

Patients undertaking peritoneal dialysis should continue to dialyse in the normal way during the administration of the oral bowel-cleansing agent. The dialysis fluid should be drained out before the procedure for which the bowel preparation has been prescribed.

\section{Renal transplant recipients}

These patients should not receive sodium phosphate preparations unless all the alternative agents are contraindicated (evidence: grade 1D).

Admission to hospital may be advisable on an individual patient basis when concerns exist over the absorption of immunosuppressants during concomitant administration of oral bowel-cleansing agents (evidence: grade 2D).

\section{Congestive cardiac failure}

Congestive cardiac failure is associated with a reduction in renal blood flow and an associated fall in GFR; the ability of these patients to excrete a phosphate load is therefore reduced, leading to an increased risk of acute phosphate nephropathy. Furthermore, these patients are at particular risk of hyponatraemia caused by the combination of hypovolaemia and high water intake.

Polyethylene glycol preparations are the preferred oral bowelcleansing agents in patients with congestive cardiac failure (evidence: grade 2D).

Patients with significant congestive cardiac failure (New York Heart Association class III or IV, or an ejection fraction below $50 \%$ ) should not receive oral sodium phosphate preparations (evidence: grade $1 \mathrm{C}$ ).

Many medications commonly prescribed to treat heart failure require evaluation before administration of an oral bowelcleansing agent. For example, where possible, diuretics, ACE inhibitors and angiotensin II receptor blockers should be discontinued in accordance with the guidance below.

\section{Liver cirrhosis and/or ascites}

Cirrhosis has been identified as a possible risk factor for acute phosphate nephropathy. Polyethylene glycol is the preferred oral 
bowel-cleansing agent for use in patients with liver cirrhosis or ascites (evidence: grade 2D).

\section{Certain medications}

ACE inhibitors and angiotensin II receptor blockers

An increase in efferent glomerular arteriolar tone is an important physiological response to hypotension and/or volume depletion, enabling the GFR to be maintained. In the presence of ACE inhibition, this compensatory response is ameliorated. Patients established on ACE inhibitors and angiotensin II receptor blockers are prone to deterioration in renal function during periods of hypovolaemia (eg, precipitated by oral bowelcleansing agents).

Furthermore, renin-angiotensin blockers also accentuate bicarbonaturia through inhibition of angiotensin II, enhancing alkalinisation of the urine. This promotes calcium and phosphate precipitation, increasing the risk of acute phosphate nephropathy in the presence of oral sodium phosphate preparations. $^{58}$

These drug effects may only pose a theoretical risk in many patients, but, where possible, renin-angiotensin blockers should be discontinued on the day of administration of oral bowelcleansing agents and not reinstated until $72 \mathrm{~h}$ after the procedure (evidence: grade $2 \mathrm{D}$ ).

\section{Diuretics}

Diuretics may alter electrolyte balance and predispose to intravascular volume depletion especially in high doses. Therefore it is advised that hydration status is assessed before administration of oral bowel-cleansing preparations in patients taking diuretics. This should include measurement of estimated GFR, but could also include clinical parameters. Low blood pressure, a fall in blood pressure on standing, dry axillae, and reduced jugular venous pressure may indicate fluid depletion, but dry mouth and reduced skin turgor can be misleading.

Unless there is judged to be a significant risk of pulmonary oedema, diuretics should be temporarily discontinued on the day of the administration of oral bowel-cleansing preparation (evidence: grade 1D).

If diuretics are continued, it is important to check electrolytes, use a polyethylene glycol preparation, and advise the patient to avoid dehydration.

\section{Non-steroidal anti-inflammatory drugs}

These medications reduce renal perfusion and therefore limit the kidneys' capacity to compensate for reduced renal perfusion through volume depletion. Where possible therefore NSAIDs should be discontinued on the day of administration of oral bowel-cleansing preparations and withheld until $72 \mathrm{~h}$ after the procedure (evidence: grade 1D).

Once daily, low-dose aspirin is commonly prescribed to patients with cardio- or cerebro-vascular disease. This medication may reasonably be continued during the administration of oral bowel-cleansing agents (evidence: Grade 1D).

\section{Medications known to induce the syndrome of inappropriate anti-} diuretic hormone secretion

These medications increase the risk of water retention and/or electrolyte imbalance, and include tricyclic antidepressants, selective serotonin reuptake inhibitors, many antipsychotic drugs and carbamazepine. While these medications need not be discontinued, serum urea and electrolytes should be checked before administration of oral bowel-cleansing preparations in patients taking them (evidence: grade 2D). The online appen- dices include a suggested template for a patient advice sheet (appendix 4) and a checklist which may help to identify the most appropriate bowel cleansing agent for any given patient (appendix 5).

\section{AREAS REQUIRING FURTHER RESEARCH}

Should the serum creatinine concentration be rechecked after a patient has received oral sodium phosphate, and when should this be undertaken?

Best practice remains unclear. Identification at a later date of nonprogressive chronic kidney disease in a typical patient who has developed acute phosphate nephropathy (an older person with hypertension and minimal proteinuria) is unlikely to provide a strong indication for renal biopsy; the link between oral bowelcleansing preparation and renal impairment is less likely to be noticed as time elapses. A decision not to check the serum creatinine concentration after oral sodium phosphate preparations may lead to cases of acute phosphate nephropathy being missed. This may result in the patient receiving further sodium phosphate preparations. The optimal timing of such a blood test has not been established. Furthermore, it is unclear whether it should be undertaken in all patients receiving oral sodium phosphate preparations or simply those at higher risk of acute phosphate nephropathy. A cost-benefit analysis is also required.

How safe is the use of oral sodium phosphate preparations in patients without those comorbidities currently identified as risk factors for acute phosphate nephropathy?

Given the current evidence base, ${ }^{59-61}$ and their superior tolerability, the use of oral sodium phosphate preparations as oral bowel-cleansing agents in patients without chronic kidney disease, congestive heart failure or liver failure probably remains acceptable. However, further studies are required to ascertain the true safety of sodium phosphate preparations as bowel-cleansing preparations for screening investigations (which, by their nature, are often repeated over time) and in patients with very early (stage 1 or 2 ) chronic kidney disease.

In the presence of predisposing conditions such as heart failure, what is the risk of acute electrolyte disorders with each preparation?

Hyponatraemia appears most likely to occur when predisposed patients drink large volumes of water, causing water intoxication as a result of overenthusiastic adherence to advice to drink 'plenty of water'. Use of polyethylene glycol preparations involves ingestion of up to 4 litres of fluid, but this is as an isotonic solution and, as such, is designed not to cause electrolyte abnormalities. However, how effective these preparations are at preventing electrolyte disorders requires further study.

Contributors All the stated authors took part in writing and editing the manuscript and have approved the final version.

Competing interests DT has spoken at sponsored meetings since the guidelines were published in draft form, but the purpose of the meetings was to explain the guidelines to clinicians. None of the authors were involved with sponsored meetings during the preparation of these guidelines

Provenance and peer review Not commissioned; externally peer reviewed.

\section{REFERENCES}

1. http://www.npsa.nhs.uk/nrls/alerts-and-directives/rapidrr/reducing-risk-of-harmfrom-oral-bowel-cleansing-solutions (accessed May 2009).

2. Wexner SD, Beck DE, Baron TH, et al; American Society of colon and Recta Surgeons; American Society for gastrointestinal Endoscopy; Society of American gastrointestinal and endoscopic Surgeons. A consensus document on bowel preparation before colonoscopy: prepared by a task force from the American Society of Colon and Rectal Surgeons (ASCRS), the American Society for gastrointestinal 
Endoscopy (ASGE), and the Society of American gastrointestinal and Endoscopic Surgeons (SAGES). Gastrointest Endosc 2006;63:894-909.

3. Guyatt $\mathbf{G H}, \mathbf{O x m a n} A D$, Kunz $\mathrm{R}$, et al. Going from evidence to recommendations. BMJ 2008;336:1049-51.

4. Jaeschke R, Guyatt GH, Dellinger $P$, et al. Use of GRADE grid to reach decisions on clinical practice guidelines when consensus is elusive. BMJ 2008;337:a744.

5. British National Formulary, London: BMJ Publishing Group Ltd and Royal Pharmaceutical Society of Great Britain, 2009.

6. El Sayed AM, Kanafani ZA, Mourad FH, et al. A randomized single-blind trial of whole versus split-dose polyethylene glycol-electrolyte solution for colonoscopy preparation. Gastrointest Endosc 2003;58:36-40.

7. Schiller LR. Clinical pharmacology and use of laxatives and lavage solutions. J Clin Gastroenterol 1999;28:11-18.

8. Vanner SJ, MacDonald PH, Paterson WG, et al. A randomized prospective trial comparing oral sodium phosphate with standard polyethylene glycol-based lavage solution (Golytely) in the preparation of patients for colonoscopy. Am J Gastroenterol 1990:85:422-7.

9. Hsu CW, Imperiale TF. Meta-analysis and cost comparison of polyethylene glycol lavage versus sodium phosphate for colonoscopy preparation. Gastrointest Endosc 1998;48:276-82.

10. Cohen SM, Wexner SD, Binderow SR, et al. Prospective, randomized, endoscopicblinded trial comparing precolonoscopy bowel cleansing methods. Dis Colon Rectum 1994:37:689-96.

11. Frommer D. Cleansing ability and tolerance of three bowel preparations for colonoscopy. Dis Colon Rectum 1997;40:100-4.

12. Kolts BE, Lyles WE, Achem SR, et al. A comparison of the effectiveness and patient tolerance of oral sodium phosphate, castor oil, and standard electrolyte lavage for colonoscopy or sigmoidoscopy preparation. Am J Gastroenterol 1993:88:1218-23.

13. Belsey J, Crosta C, Epstein 0, et al. Meta-analysis: the relative efficacy of oral bowel preparations for colonoscopy 1985-2010. Aliment Pharmacol Ther 2012; $35: 222-37$

14. Nelson DB, Barkun AN, Block KP, et al. Technology status evaluation report. Colonoscopy preparations. Gastrointest Endosc 2001;54:829-32.

15. Hoy SM, Scott LJ, Wagstaff AJ. Sodium picosulfate/magnesium citrate: a review of its use as a colorectal cleanser. Drugs 2009;69:123-36.

16. Miki P Jr, Lemos CR, Popoutchi P, et al. Comparison of colon-cleansing methods in preparation for colonoscopy-comparative efficacy of solutions of mannitol, sodium picosulfate and monobasic and dibasic sodium phosphates. Acta Cir Bras 2008;23 (Suppl 1):108-11.

17. Renaut AJ, Raniga S, Frizelle FA, et al. A randomized controlled trial comparing the efficacy and acceptability of phospo-soda buffered saline (Fleet) with sodium picosulphate/magnesium citrate (Picoprep) in the preparation of patients for colonoscopy. Colorectal Dis 2008;10:503-5.

18. Tjandra JJ, Chan M, Tagkalidis PP. Oral sodium phosphate (Fleet) is a superior colonoscopy preparation to Picoprep (sodium picosulfate-based preparation). Dis Colon Rectum 2006;49:616-20.

19. Schmidt LM, Williams P, King D, et al. Picoprep-3 is a superior colonoscopy preparation to Fleet: a randomized, controlled trial comparing the two bowel preparations. Dis Colon Rectum 2004;47:238-42.

20. Yoshioka K, Connolly AB, Ogunbiyi OA, et al. Randomized trial of oral sodium phosphate compared with oral sodium picosulphate (Picolax) for elective colorectal surgery and colonoscopy. Dig Surg 2000;17:66-70.

21. Hamilton D, Mulcahy D, Walsh D, et al. Sodium picosulphate compared with polyethylene glycol solution for large bowel lavage: a prospective randomised trial. Br J Clin Pract 1996:50:73-5.

22. Macleod AJ, Duncan KA, Pearson RH, et al. A comparison of fleet Phospho-soda with Picolax in the preparation of the colon for double contrast barium enema. Clin Radiol 1998;53:612-14.

23. Taylor SA, Halligan S, Goh V, et al. Optimizing bowel preparation for multidetector row CT colonography: effect of Citramag and Picolax. Clin Radiol 2003;58:723-32.

24. Lai AK, Kwok PC, Man SW, et al. A blinded clinical trial comparing conventional cleansing enema, Pico-salax and Golytely for barium enema bowel preparation. Clin Radiol 1996;51:566-9

25. Swarbrick MJ, Collins MC, Moore DJ, et al. A comparative trial of magnesium citrate (Citramag) and Picolax for barium enema bowel preparation. Clin Radiol 1994:49:379-81.

26. Mc Laughlin P, Eustace J, Mc Sweeney S, et al. Bowel preparation in CT colonography: electrolyte and renal function disturbances in the frail and elderly patient. Eur Radiol 2010:20:604-12.

27. Bowles CJ, Leicester R, Romaya C, et al. A prospective study of colonoscopy practice in the UK today: are we adequately prepared for national colorectal cancer screening tomorrow? Gut 2004:53:277-83.

28. Vradelis S, Kalaitzakis $E$, Sharifi $Y$, et al. Addition of senna improves quality of colonoscopy preparation with magnesium citrate. World J Gastroenterol 2009;15:1759-63

29. Kastenberg D, Chasen R, Choudhary C, et al. Efficacy and safety of sodium phosphate tablets compared with PEG solution in colon cleansing: two identically designed, randomized, controlled, parallel group multicenter phase III trials. Gastrointest Endosc 2001;54:705-13.

30. Eschinger EJ, Littman JJ, Meyer $\mathrm{K}$, et al. Safety of sodium phosphate tablets in patients receiving propofol-based sedation for colonoscopy. J Clin Gastroenterol 2004;38:425-8.
31. Reddy DN, Rao GV, Sriram PV. Efficacy and safety of oral sodium phosphate versus polyethylene glycol solution for bowel preparation for colonoscopy. Indian J Gastroenterol 2002:21:219-21.

32. Brunelli SM, Feldman HI, Latif SM, et al. A comparison of sodium phosphosoda purgative to polyethylene glycol bowel preparations prior to colonoscopy. Fam Med 2009;:41:39-45.

33. Beloosesky Y, Grinblat J, Weiss A, et al. Electrolyte disorders following oral sodium phosphate administration for bowel cleansing in elderly patients. Arch Intern Med 2003:163:803-8.

34. Ainley EJ, Winwood PJ, Begley JP. Measurement of serum electrolytes and phosphate after sodium phosphate colonoscopy bowel preparation: an evaluation. Dig Dis Sci 2005:50:1319-23.

35. Ho JM, Juurlink DN, Cavalcanti RB. Hypokalemia following polyethylene glycol-based bowel preparation for colonoscopy in older hospitalized patients with significant comorbidities. Ann Pharmacother 2010;44:466-70.

36. Lien YH. Is bowel preparation before colonoscopy a risky business for the kidney? Nat Clin Pract Nephrol 2008:4:606-14.

37. Tjandra JJ, Tagkalidis P. Carbohydrate-electrolyte (E-Lyte) solution enhances bowel preparation with oral fleet phospho-soda. Dis Colon Rectum 2004;47:1181-6.

38. Frizelle FA, Colls BM. Hyponatremia and seizures after bowel preparation: report of three cases. Dis Colon Rectum 2005;48:393-6.

39. Nagler J, Poppers D, Turetz M. Severe hyponatremia and seizure following a polyethylene glycol-based bowel preparation for colonoscopy. J Clin Gastroenterol 2006;40:558-9.

40. Nyberg C, Hendel J, Nielsen $\mathrm{OH}$. The safety of osmotically acting cathartics in colonic cleansing. Nat Rev Gastroenterol Hepatol 2010;7:557-64.

41. Markowitz GS, Radhakrishnan J, D'Agati VD. Towards the incidence of acute phosphate nephropathy. J Am Soc Nephrol 2007;18:3020-2.

42. Markowitz GS, Stokes MB, Radhakrishnan J, et al. Acute phosphate nephropathy following oral sodium phosphate bowel purgative: an underrecognized cause of chronic renal failure. J Am Soc Nephrol 2005;16:3389-96.

43. Gumurdulu Y, Serin E, Ozer B, et al. Age as a predictor of hyperphosphataemia after oral phosphosoda administration for colon preparation. $J$ Gastroenterol Hepatol 2004:19:68-72.

44. Hookey LC, Vanner S. Recognizing the clinical contraindications to the use of oral sodium phosphate for colon cleansing: a case study. Can J Gastroenterol 2004; 18:455-8.

45. Brunelli SM, Lewis JD, Gupta M, et al. Risk of kidney injury following oral phosphosoda bowel preparations. J Am Soc Nephrol 2007:18:3199-205.

46. Sica DA, Carl D, Zfass AM. Acute phosphate nephropathy -an emerging issue. Am J Gastroenterol 2007;102:1844-7.

47. Food and Drug Administration. Oral Sodium Phosphate (OSP) Products for Bowel Cleansing. 2006. http://www.fda.gov/cder/drug/infopage/osp_solution laccessed 11 May 2009).

48. Kontani M, Hara A, Ohta S, et al. Hypermagnesemia induced by massive cathartic ingestion in an elderly woman without pre-existing renal dysfunction. Intern Med 2005:44:448-52.

49. Onishi S, Yoshino S. Cathartic-induced fatal hypermagnesemia in the elderly. Intern Med 2006;45:207-10.

50. Schelling JR. Fatal hypermagnesemia. Clin Nephrol 2000;53:61-5.

51. Jung B, Påhlman L, Nyström PO; Mechanical Bowel Preparation Study Group. Multicentre randomized clinical trial of mechanical bowel preparation in elective colonic resection. Br J Surg 2007:94:689-95.

52. Contant CM, Hop WC, van't Sant HP, et al. Mechanical bowel preparation for elective colorectal surgery: a multicentre randomised trial. Lancet 2007:370:2112-17.

53. Rex DK. Dosing considerations in the use of sodium phosphate bowel preparations for colonoscopy. Ann Pharmacother 2007;41:1466-75.

54. Rostom A, Jolicoeur E, Dubé C, et al. A randomized prospective trial comparing different regimens of oral sodium phosphate and polyethylene glycol-based lavage solution in the preparation of patients for colonoscopy. Gastrointest Endosc 2006;64:544-52

55. Powell-Tuck J, Gosling P, Lobo DN, et al. British Consensus Guidelines on Intravenous Fluid Therapy for Adult Surgical Patients. GIFTASUP. http://www.renal. org/pages/media/Guidelines/GIFTASUP\%20FINAL_31-10-08.pdf laccessed 10 Aug 2009)

56. Vilien M, Rytkønen M. Golytely preparation for colonoscopy: 1.5 liters is enough for outpatients. Endoscopy 1990;22:168-70.

57. Barclay RL, Depew WT, Vanner SJ. Carbohydrate-electrolyte rehydration protects against intravascular volume contraction during colonic cleansing with orally administered sodium phosphate. Gastrointest Endosc 2002;56:633-8.

58. Beyea A, Block C, Schned A. Acute phosphate nephropathy following oral sodium phosphate solution to cleanse the bowel for colonoscopy. Am J Kidney Dis 2007;50:151-4.

59. Russmann S, Lamerato L, Marfatia A, et al. Risk of impaired renal function after colonoscopy: a cohort study in patients receiving either oral sodium phosphate or polyethylene glycol. Am J Gastroenterol 2007;102:2655-63.

60. Singal AK, Rosman AS, Post JB, et al. The renal safety of bowel preparations for colonoscopy: a comparative study of oral sodium phosphate solution and polyethylene glycol. Aliment Pharmacol Ther 2008:27:41-7.

61. Hurst FP, Bohen EM, Osgard EM, et al. Association of oral sodium phosphate purgative use with acute kidney injury. J Am Soc Nephrol 2007;18:3192-8. 